Les moments d'observation sont corrigés pour l'aberration. Deux observations faites à Kremsmünster le 15 et 23 Juin donnaient plusieurs minutes de différence pour chaque coordonnée.

Au moyen de cette comparaison je parvins aux six positions normales que voici:

\begin{tabular}{|c|c|c|c|c|c|}
\hline \multirow[b]{3}{*}{ I. } & \multicolumn{3}{|c|}{ Erreurs des éléments provis. } & \multicolumn{2}{|c|}{ Positions normales } \\
\hline & Temps. & $\Delta \alpha \cos \delta$ & $\Delta \delta$ & $\mathbf{A R}$ & Decl. \\
\hline & Juin 1.5 & $-8^{\prime \prime} 6$ & $-5^{\prime \prime} 3$ & $13^{\circ} 6^{\prime} 34^{\prime \prime} 1$ & $+28^{\circ} 23^{\prime} 3^{\prime \prime} 8$ \\
\hline II. & 6.5 & -3.3 & +1.7 & $1440 \quad 44.3$ & +265614.1 \\
\hline III. & 14.5 & +0.3 & +3.1 & $\begin{array}{lll}17 & 34 & 58.2\end{array}$ & $+2351 \quad 18.9$ \\
\hline IV. & 20.5 & +0.2 & -2.2 & $20 \quad 18 \quad 13.8$ & $+203337 . \tilde{3}$ \\
\hline $\mathrm{V}$ & Juillet 1.5 & +3.1 & +24.9 & $\begin{array}{lll}27 & 51 & 55.9\end{array}$ & +95721.4 \\
\hline VI. & 7.5 & -8.0 & +61.4 & $\begin{array}{lll}35 & 0 & 0.9\end{array}$ & -1315.8 \\
\hline
\end{tabular}

En me fondant sur les positions de Juin 1.5 et Juillet 7.5 et en variant les distances de la terre, j'ai enfin obtenu les valeurs suivantes pour les éléments les plus probables.

Comète I. 1870 .

$T=1870$ Juillet 14.07762 (t. m. de Greenwich)

$$
\left.\begin{array}{rlrl}
\pi & =303^{\circ} 32^{\prime} 11^{\prime \prime} 2 \\
\delta & =141 & 44 & 52.2 \\
i & =58 & 12 & 29.5
\end{array}\right\} \begin{gathered}
\text { Equinoxe moyen } \\
1870.0
\end{gathered}
$$

$\log q=0.0037703$

Mouvement rétrograde.

Les positions - normales sont ainsi satisfaites par le calcul :

$$
\begin{aligned}
& \text { Juin } 1.5 \underbrace{\Delta \lambda \cos \beta}_{+0^{\prime \prime} 1} \frac{\Delta \beta}{0^{\prime \prime} 0} \\
& 6.5+3.4+3.8 \\
& 14.5+1.8+3.0 \\
& 20.5-5.1-4.1 \\
& \text { Juillet } 1.5+1.5+1.4 \\
& 7.5+0.7+0.1
\end{aligned}
$$

La somme des earrés des erreurs, qui auparavant était $4593^{\prime \prime} 99$, se trouve réduite à $85^{\prime \prime} 78$.

Le calcul étant entièrement achevé, j'aperçus dans les volumes LXXVII. - LXXVIII. des A. N. quelques observations de la comète, dont la publication a été retardée. La comparaison avec l'orbite définitive m'a donné les erreurs suivantes:

$$
\begin{aligned}
& \underbrace{\text { T. m. de Greenw. }}_{\text {Mai } 30.52193} \underbrace{\Delta \alpha}_{+10^{\prime \prime} 5} \underbrace{\Delta \delta}_{+1^{\prime \prime 2}} \text { Vienne. } \\
& \text { Juin } 5.53244-0.2+6.5 \quad \text { Bonn. } \\
& 5.54154-2.7+4.8 \quad- \\
& \begin{array}{llll}
6.50791 & -9.3 & -2.4 & - \\
6.51323 & +7.6 & +0.3 & \text { Hambourg. }
\end{array} \\
& 6.52590-2.8+5.8 \text { Bonn. } \\
& 7.51902+0.1+3.9- \\
& 7.52961+5.8+4.7- \\
& 8.53402+14.7-2.6 \quad \text { Vienne. } \\
& 22.49247+5.3-28.6 \quad-
\end{aligned}
$$

Ayant accompli tous ces calculs, j’ai enfin trouvé les résultats d'un travail semblable, pour la même comète, dans les Comptes Rendus de l'Acadèmie de Vienne (Sitzungsberichte, math.-naturw. Klasse, LXIV., 2, 1871, p. 147). Les éléments définitifs, auxquels M. A. Seydler est parvenu, et qui ne sont pas encore mentionnés dans ce journal, concordent avec les miens d'une manière très-satisfaisante. Effectivement la comparaison donne ces différences assez insignifiantes:

$$
\begin{aligned}
\Delta T & =-0.00456 \\
\Delta \pi & =+26^{\prime \prime} 2 \\
\Delta \delta & =+4.8 \\
\Delta i & =+21.9 \\
\Delta \log q & =+0.0000118
\end{aligned}
$$

L'on reconnait, par cette comparaison, le degré d'exactitude, avec lequel les éléments de cette orbite ont pu être déterminés, malgré le petit nombre d'observations.

Copenhague, le 30 Sept. $1872 . \quad J$. Dreyer.

\title{
Beobachtungen, Elemente und Ephemeride des Planeten (123).
}

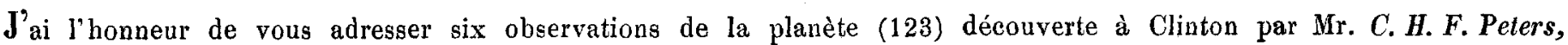
le 31 Juillet dernier, ainsi'que des éléments de l'orbite et une courte éphéméride de cette planète. Les éléments sont déduits des observations de Clinton des 1 et 23 Aout qui ont été publiées dans les Astr. Nachr. et de l'observation de Marseille du 17 Septembre. 
Observations de la planète (123).

Date de Heure de l'ohserv.

* de Comparaison. l'observation. t. m. de Marseille.

$a$
$a$
$a$
$b$
$b$
$b$

\begin{tabular}{|c|c|c|}
\hline \multicolumn{2}{|c|}{1872 Sept. 17} & $10^{\mathrm{h}} 58^{\mathrm{m}} 4^{\mathrm{s}}$ \\
\hline & $\rtimes 20$ & 111358 \\
\hline & $\gg 21$ & $\begin{array}{lll}10 & 19 & 13\end{array}$ \\
\hline & ᄁ 29 & $\begin{array}{lll}9 & 45 & 10\end{array}$ \\
\hline & $>30$ & $\begin{array}{lll}9 & 4218\end{array}$ \\
\hline & Oet. 1 & $\begin{array}{lll}9 & 21 & 3\end{array}$ \\
\hline
\end{tabular}

\begin{tabular}{|c|c|c|c|}
\hline AR de (123) & g f. par. & P. de $(123)$ & $\log f$. par. \\
\hline $21^{\mathrm{h}} 19^{\mathrm{m}} 57^{\mathrm{s}} 44$ & 15 & $101^{0} 41^{\prime} 34^{\prime \prime} 8$ & 586 \\
\hline 211836.18 & +1 & $10144 \quad 42.4$ & 527 \\
\hline $21 \quad 18 \quad 13.35$ & +1.096 & $101 \quad 45 \quad 34.6$ & 604 \\
\hline $21 \quad 15 \quad 58.16$ & +1 . & $101 \quad 49 \quad 26.4$ & -0 \\
\hline $21 \quad 1548$ & +1 . & $\begin{array}{lll}101 & 49 & 30.3\end{array}$ & -0 \\
\hline $\begin{array}{lll}1 & 15 & 40.14\end{array}$ & +2.944 & 1014928.5 & -0.6976 \\
\hline
\end{tabular}

Positions moyennes des étoiles de comparaison pour 1872,0 .

$$
\begin{array}{rrlr}
a & 41698 \text { Lal. Cat. } \quad \mathrm{AR}=21^{\mathrm{h}} 20^{\mathrm{m}} 34^{\mathrm{s}} 28, & \mathrm{P} .=101^{\circ} 43^{\prime} 1^{\prime \prime} 8 \\
b & 280 \text { Weisse H. XXI. } & 211352.60 & 1015326.7
\end{array}
$$

Eléments de l'orbite de la planète (123).

Epoque 1872 Septembre 17,46849 (temps moyen de Berlin)

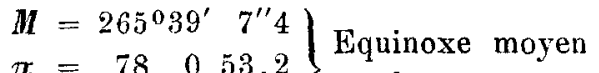

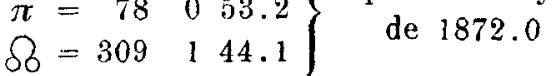

$$
\begin{aligned}
& i=63836.2 \\
& \varphi=61421.1 \\
& \mu=804^{\prime \prime} 118
\end{aligned}
$$

\begin{tabular}{|c|c|c|c|}
\hline T. $\mathrm{m}$. & de Berlin. & AR de (123) & P. de (123) \\
\hline 1872 & Oct. 26.5 & $21^{\mathrm{h}} 20^{\mathrm{m}} 17^{\mathrm{s}}$ & $101^{\circ} 18^{\prime} 7^{\prime \prime}$ \\
\hline & 27.5 & $\begin{array}{lll}21 & 20 & 47\end{array}$ & $101 \quad 1536$ \\
\hline & 28.5 & $2121 \quad 18$ & $101 \quad 12 \quad 59$ \\
\hline & 29.5 & $\begin{array}{lll}21 & 21 & 51\end{array}$ & $101 \quad 10 \quad 16$ \\
\hline & 30.5 & $\begin{array}{lll}21 & 22 & 24\end{array}$ & $101 \quad 727$ \\
\hline & 31.5 & $\begin{array}{lll}21 & 22 & 59\end{array}$ & $101 \quad 432$ \\
\hline & Nov, 1.5 & $2123 \quad 35$ & $101+31$ \\
\hline & 2.5 & $2124 \quad 13$ & $100 \quad 58 \quad 24$ \\
\hline
\end{tabular}$$
\log a=0.429791
$$

Ephéméride de la planete (123).
1872 Nov. 3.5

4.5

$\underbrace{\text { AR de (123) }}_{21^{h}\left(24^{m} 1^{s}\right.}$

$21^{\mathrm{h}} 24^{\mathrm{m}} 51^{\mathrm{s}}$

1

$\begin{array}{llll}5.5 & 21 & 26 & 12\end{array}$

$\begin{array}{llll}6.5 & 21 & 26 & 54\end{array}$

$\begin{array}{llll}7.5 & 21 & 27 & 37\end{array}$

$\begin{array}{llll}8.5 & 21 & 28 & 22\end{array}$

$\begin{array}{llll}9.5 & 21 & 29 & 7\end{array}$

10.5

$21 \quad 29 \quad 54$

11.5

$2130 \quad 41$

12.5

213130

13.5

$\begin{array}{lll}21 & 32 & 19\end{array}$

14.5

$2133 \quad 10$

15.5

$\begin{array}{lll}21 & 34 & 1\end{array}$

16.5

17.5

18.5

$2134 \quad 54$

$\begin{array}{lll}21 & 35 & 47\end{array}$

0.3645

19.5

$\begin{array}{lll}21 & 36 & 42\end{array}$

$21 \quad 37 \quad 37$

Marseille, 1872 Octobre 26 . $\log \Delta$

0.3736

1005154

$10048 \quad 30$

$\begin{array}{lll}100 & 45 \quad 1\end{array}$

1004126

1003745

$100 \quad 33 \quad 59$

$10030 \quad 7$

$\begin{array}{lll}100 & 26 & 9\end{array}$

$100 \quad 22 \quad 6$

$100 \quad 17 \quad 57$

$\begin{array}{lll}100 & 13 \quad 43\end{array}$

$\begin{array}{lll}100 \quad 9 & 23\end{array}$

$100 \quad 4 \quad 58$

$\begin{array}{lll}100 & 0 & 27\end{array}$

$\begin{array}{lll}99 & 55 & 51\end{array}$

$\begin{array}{lll}99 & 51 \quad 8\end{array}$

0.4087

Literarische Anzeige.

In dem unterzeichneten Verlage erschien soeben:

\section{Mittlere Oerter für 1872,0 von 539 Sternen,}

und scheinbare Oerter für das Jahr 1872 von 529 Sternen des Verzeichnisses I. und II., welches nach der Vierteljahrsschrift der, Astronomischen Gesellschaft", IV. Jahrgang, 4. Heft 1869 , für die Beobachtung der Sterne der nördlichen Halbkugel bis zur neunten Grösse als Grundlage dienen soll. Unter Mitwirkung der "Astronomischen Ge- sellschaft" herausgegeben von der Redaction des Berliner Astronomischen Jahrbuches. gr. 8. geh. $15 \mathrm{Sgr}$.

Die beiden früheren Jahrgänge sind zum gleichen Preise (je 15 Sgr.) zu erhalten.

Von jeder Buchhandlung ist unentgeltich zu erhalten ein Verzeichniss von astronomischen, meteorologischen und geodätischen Schriften unsers Verlages, auch direct auf frankirte Bestellung. Ferd. Dümmler's Verlagsbuchhandlung, Harrwitz of Gossmann in Berlin.

Altona 1872 . November 9.

Herausgeber: Prof. C. A. F. Petors, Altona, Palmaille ㅑㅛ 12. - Druck von Gustav Esch in Altona. 